\title{
A Media and Clinic Intervention to Increase Colorectal Cancer Screening in Ohio Appalachia
}

\author{
Jessica L. Krok-Schoen, ${ }^{1}$ Mira L. Katz, ${ }^{1,2,3}$ Jill M. Oliveri, ${ }^{1}$ Gregory S. Young, \\ Michael L. Pennell, ${ }^{5}$ Paul L. Reiter, ${ }^{1,2}$ Jesse J. Plascak, ${ }^{6}$ \\ Michael D. Slater, ${ }^{7}$ Janice L. Krieger, ${ }^{8}$ Cathy M. Tatum, ${ }^{1}$ and Electra D. Paskett ${ }^{1,2,9}$ \\ ${ }^{1}$ Comprehensive Cancer Center, The Ohio State University, 1590 N. High Street, Suite 525, Columbus, OH 43201, USA \\ ${ }^{2}$ Division of Cancer Prevention and Control, Department of Internal Medicine, College of Medicine, The Ohio State University, \\ 395 W. 12th Avenue, Columbus, OH 43210, USA \\ ${ }^{3}$ Division of Health Behavior and Health Promotion, College of Public Health, The Ohio State University, 1841 Neil Avenue, \\ Columbus, OH 43210, USA \\ ${ }^{4}$ Center for Biostatistics, The Ohio State University, 1800 Cannon Drive, Columbus, OH 43210, USA \\ ${ }^{5}$ Division of Biostatistics, College of Public Health, The Ohio State University, 1841 Neil Avenue, Columbus, OH 43210, USA \\ ${ }^{6}$ Biobehavioral Cancer Prevention and Control Training Program, University of Washington, P.O. Box 359455, Seattle, WA 98195, USA \\ ${ }^{7}$ School of Communication, The Ohio State University, 3022 Derby Hall, 154 N. Oval Mall, Columbus, OH 43210, USA \\ ${ }^{8}$ STEM Translational Communication Program, University of Florida, 2088 Weimer Hall, 1885 Stadium Road, Gainesville, \\ FL 32611, USA \\ ${ }^{9}$ Division of Epidemiology, College of Public Health, The Ohio State University, 1841 Neil Avenue, Columbus, OH 43210, USA
}

Correspondence should be addressed to Electra D. Paskett; electra.paskett@osumc.edu

Received 13 March 2015; Revised 29 June 2015; Accepted 16 August 2015

Academic Editor: Anne Miles

Copyright (C) 2015 Jessica L. Krok-Schoen et al. This is an open access article distributed under the Creative Commons Attribution License, which permits unrestricted use, distribution, and reproduction in any medium, provided the original work is properly cited.

Objective. To test the effectiveness of a colorectal cancer (CRC) screening intervention among adults living in Ohio Appalachia. Methods. We conducted a group-randomized trial of a county-level intervention among adults living in 12 Ohio Appalachian counties who received a media campaign and clinic intervention focused on either CRC screening or fruits and vegetables. Participants' percentage within CRC screening guidelines was assessed with cross-sectional surveys conducted annually for four years, and validated with medical record review of screening. Results. On average, screening data were obtained on 564 intervention and 559 comparison participants per year. There was no difference in the Wave 4 CRC screening rates of intervention and comparison counties (35.2\% versus $31.4 \%$ ). Multivariate analyses found that high perceived risk of CRC, willingness to have a CRC test if recommended by a doctor, doctor recommendation of a CRC screening test, and patient-physician communication about changes in bowel habits, family history of CRC, and eating fruits and vegetables were significant $(p<0.05)$ predictors of being within CRC screening guidelines. Conclusions. The intervention was not effective in increasing CRC rates among Ohio Appalachian adults. Future research should determine how media and clinic-based interventions can be modified to improve CRC screening rates among this underserved population.

\section{Introduction}

Colorectal cancer (CRC) is the third leading type of cancer and the second leading cause of cancer death among men and women in the United States [1]. Significant disparities in incidence, mortality, and survival rates exist among underserved populations for this disease [1-6]. Moreover, CRC screening modalities are less likely to be used regularly among underserved populations [4-6]. One underserved population that bears an excess burden of CRC is residents of Ohio Appalachia, a 32-county region in southern and eastern Ohio [6]. Rates of CRC incidence and mortality are 
approximately $17 \%$ and $18 \%$ higher, respectively, among Ohio Appalachian residents compared to the average age-adjusted US population in $2005[7,8]$. Many factors common among residents living in Ohio Appalachia may contribute to CRC disparities, particularly limited access to cancer screening, low socioeconomic status (SES), and behavioral factors (poor diet, increased tobacco use) $[2,7,9]$.

Screening and early detection have the potential to significantly reduce CRC incidence and mortality [10]. Previous media campaigns about CRC screening have resulted in greater reported exposure to messages about CRC screening $[11,12]$, increased intention to speak to doctors about CRC screening [12], and increased CRC screening [13, 14] at a relatively low cost per person screened [14]. The goal of this study was to implement and evaluate a county-level intervention consisting of media and clinic-level components to increase CRC screening in Ohio Appalachian residents.

\section{Materials and Methods}

This study used a group-randomized trial design. A CONSORT diagram (Figure 1) outlines the study design. Twelve Ohio Appalachian counties were stratified into three groups (high, medium, and low) based on the average percent of late stage CRC diagnoses (obtained from the Ohio Cancer Information Surveillance System). Within each of the groups, the four counties were randomized to either the intervention or comparison condition. Details regarding the development and design of the CRC screening intervention have been previously reported $[15,16]$; however, we briefly describe the intervention components below.

2.1. Study Design. The effect of the intervention program, "Get Behind Your Health! Talk to Your Doctor About Colon Cancer Screening," on CRC screening rates was evaluated, using telephone surveys of randomly selected residents in each of the 12 counties. Surveys were conducted annually for four years over a four-month period each year (preintervention (Wave 1), postmedia only (Wave 2), postclinic intervention only (Wave 3), and postcombination media and clinic intervention (Wave 4)), with medical record review (MRR) for those who reported completing a CRC screening test to validate self-reports of CRC screening behavior (fecal occult blood test (FOBT), flexible sigmoidoscopy, or colonoscopy).

The study was powered for a mixed model ANCOVA analysis [17] based on a comparison of Wave 4 screening rates. It was estimated that a sample size of 90 participants per county was needed to achieve $80 \%$ power to detect a difference of $10 \%$ in screening rates assuming a screening rate of $32 \%$ in the comparison arm (based on Ohio BRFSS data) and an Intraclass Correlation Coefficient (ICC) of 0.0046. Based on this calculation, 6 counties were randomized to each condition (intervention and comparison) and 90 residents from each county were recruited for each survey.

2.2. Eligibility and Recruitment. The following methodology was used for each wave of data collection. Names of residents in each of the 12 Ohio Appalachian counties were randomly selected from InfoUSA County Directories. Names were sampled with replacement at each wave. Potentially eligible participants were mailed a study packet that included a recruitment letter and a study information handout outlining key elements of standard informed consent and HIPAA authorization documents. Five days after the letters were sent, trained interviewers called potential participants and described the study, addressed concerns, answered questions, and assessed eligibility. Verbal informed consent and HIPAA authorization were obtained, and then the cross-sectional survey was administered.

Participants were eligible if they (1) were men and women aged 51-75 years; (2) had a working phone number; (3) were English-speaking and able to give informed consent; (4) were a resident of one of the 12 Ohio Appalachian counties at the time of the interview; (5) had no prior history of invasive cancer (including CRC), polyps, inflammatory bowel disease, Crohn's disease, or colitis; and (6) had no strong family history of polyps, CRC, or hereditary CRC syndromes. Participants received a $\$ 10$ gift card after completing each survey as an expression of appreciation for their time. A medical record release form was also sent to each participant with a postage-paid envelope to return the completed form to the study office. Informed consent procedures and the study protocol were approved by the Institutional Review Board of The Ohio State University.

2.3. Intervention. The "Get Behind Your Health! Talk to Your Doctor About Colon Cancer Screening" intervention utilized a community-based participatory research approach to develop and pilot the CRC screening intervention. Based on results from a community assessment and in partnership with community members from Ohio Appalachia, a culturally sensitive media campaign that focused on increasing CRC screening was developed for use in the current study [15].

The intervention consisted of two main components: (1) a media campaign and (2) a clinic intervention $[15,16]$. The intervention was based on health behavior theories, including the Health Belief Model (HBM) [18], the Theory of Reasoned Action (TRA) [19], Social Cognitive Theory (SCT) [20, 21], and Attitude Accessibility Theory (AAT) [22]. The theoretical constructs included in the campaign were as follows: HBM, which helped identify perceived benefits and barriers associated with CRC screening; TRA, which helped identify specific beliefs that must be reinforced or countered (e.g., community members talk about media campaigns, influencing social norms); SCT, which provided a structure for creating messages that model desirable behaviors and teach skills necessary to enact the behaviors (e.g., "Talk to your doctor about CRC screening"); and AAT, which suggested that messages should be proximal to opportunities to enact behavior for maximum impact (e.g., clinic-based reminders).

2.4. Media Campaign Intervention. The media campaign was conducted in the six intervention counties in Waves 2 and 4 of the study. The campaign in each county featured countyspecific CRC survivors, individuals who had completed CRC 


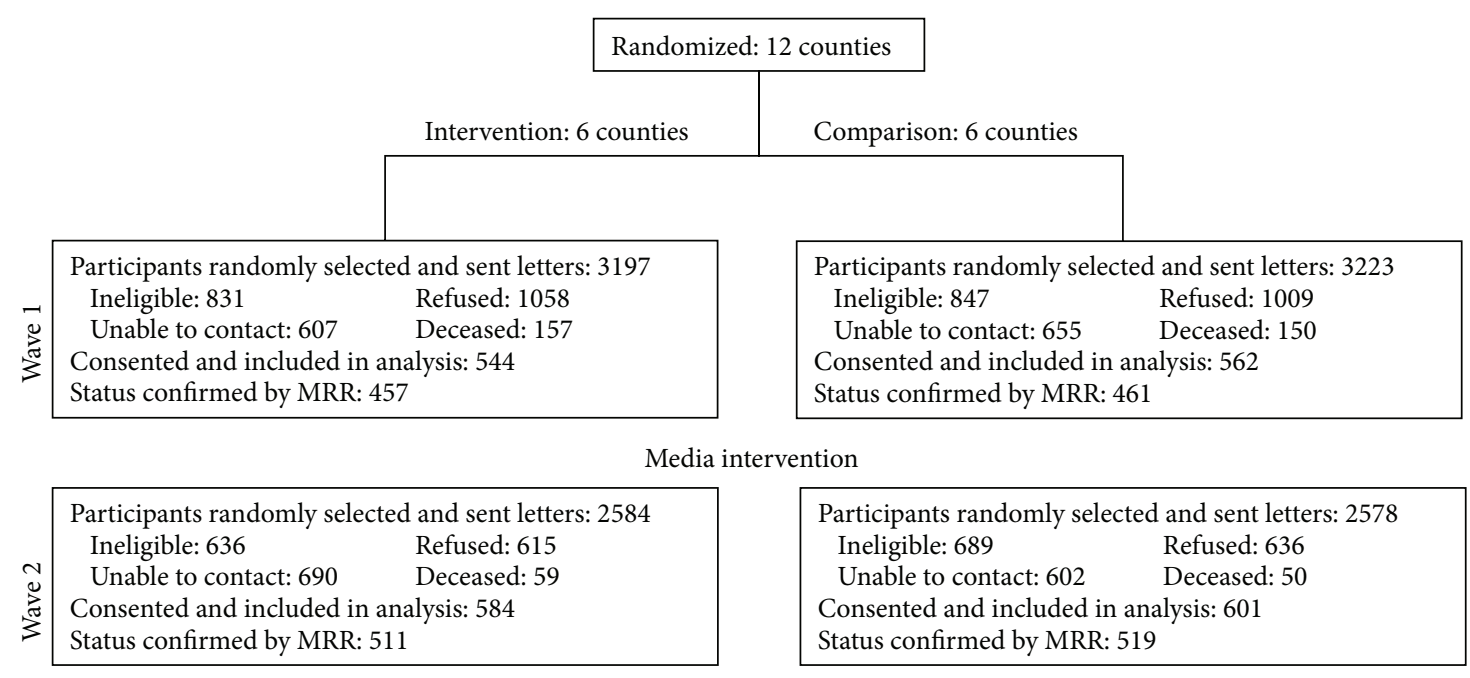

Clinic intervention

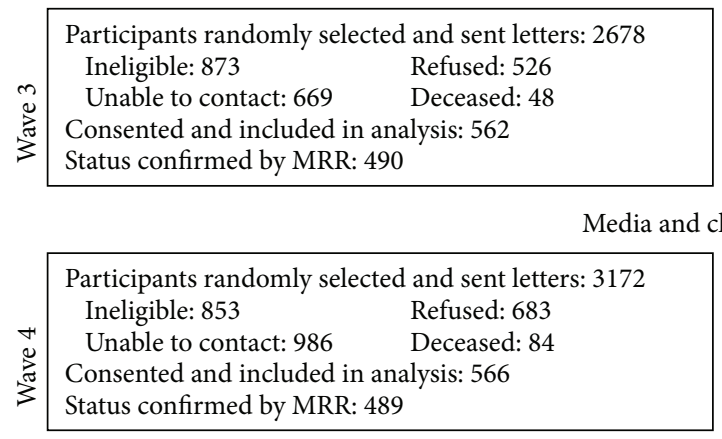

\begin{tabular}{|l|l|}
\hline Participants randomly selected and sent letters: 2671 \\
Ineligible: $818 \quad$ Refused: 466 \\
Unable to contact: $771 \quad$ Deceased: 69 \\
Consented and included in analysis: 547 \\
Status confirmed by MRR: 457 \\
\hline
\end{tabular}

tervention

Participants randomly selected and sent letters: 3194
Ineligible: $858 \quad$ Refused: 693
Unable to contact: $1032 \quad$ Deceased: 86
Consented and included in analysis: 525
Status confirmed by MRR: 448

FiguRE 1: CONSORT diagram.

screening, local physicians/nurses, and community members who were selected by the local community cancer coalition. The campaign images and messages were used in all campaign materials including billboard, posters, and articles sent to local newspapers. Although the billboard information was limited (slogan and photos of community members), the posters and newspaper articles included information about CRC, CRC risk factors and symptoms, CRC screening, and the message that CRC screening saves lives [15]. The billboard and posters were placed in high volume areas near the geographic center of each county, as determined by the local community cancer coalitions.

2.5. Clinic Intervention. Clinics within the six counties randomized to receive the intervention also received an intervention that included American Cancer Society (ACS) CRC educational posters and brochures in Wave 3 through the end of Wave 4 of the study. Clinic posters and brochures provided information about the mortality rates for CRC and motivational messages such as "If you're over 50 , you need to get tested for colon cancer" and "Talk to your doctor about getting tested for colon cancer." Local clinic managers were asked to display the ACS CRC materials in high visibility areas in waiting areas and exam rooms.
2.6. Comparison Group. The six comparison counties received a media campaign and patient education material in clinics related to healthy eating, "PEACHES" (Promoting Education in Appalachia on Cancer and Healthy Eating Styles), at the same time points the CRC screening intervention occurred in the intervention counties in Waves 2 through 4 of the study. The PEACHES campaign in each county featured local community members and farmers who were selected by the local community cancer coalition.

2.7. Study Timeline. The baseline participant recruitment began in September 2009 and was completed in April 2010. The media component of the intervention began in August 2010 and finished in July 2011. The clinic-based component of the intervention began in August 2011 and finished in July 2012. The combined intervention (i.e., media and clinic components) began in August 2012 and finished in July 2013. The final cross-sectional surveys (Wave 4) and final MRR were completed by December 2013.

2.8. Measures. The primary outcome was whether the participants were within the current U.S. Preventive Services Task Force (USPSTF) CRC screening guidelines for adults ages 50-75 (e.g., completed either an annual FOBT, a flexible sigmoidoscopy in the past five years combined with FOBT 
within the past three years, or a colonoscopy in the past 10 years) [10] as determined by MRR. The main independent variable was whether each participant lived in an intervention or comparison county.

\subsection{Independent Variables}

2.9.1. Participant Demographic Characteristics. Participants provided information about their age, gender, race, ethnicity, marital status, education, household income, employment status, and health insurance.

2.9.2. Participant Healthcare. Participants were asked about their comorbidities, CRC screening history, regular sources of medical care, and most recent CRC test (where the test and results were obtained).

2.9.3. Perceived CRC Risk. To assess CRC risk, participants were asked "Compared to other men/women your age, what do you think your risk of getting colon cancer is in your lifetime?" Response was on a 5-point Likert scale (much lower, somewhat lower, about the same, somewhat higher, and much higher) [20]. Responses were dichotomized into high perceived risk (i.e., somewhat higher and much higher) and low perceived risk (i.e., much lower, somewhat lower, and about the same).

2.9.4. Intention to Screen. Participants were asked (yes/no) if they were willing to have a CRC screening test if recommended by their doctor, have thought about talking to their doctor about completing a CRC screening test in the next year, intended to complete a CRC screening test in the next 6 months, and asked their doctor to order a CRC screening test.

2.9.5. Participant-Reported Physician Actions regarding CRC and CRC Screening. Participants were asked (yes/no) if their doctor ever asked them about eating more fruits and vegetables, their family history of CRC, changes in bowel habits, rectal bleeding, and having a CRC screening test.

2.10. Process Evaluation. Process evaluation in Wave 2 involved a subset of participants (80 adults per county) responding to the mail-in survey asking if they had seen the campaign messages (billboards/posters used in the media campaign) during the past year. In order to ensure correct identification of campaign messages, pictures of fictitious CRC screening campaigns and similar questions addressing the sham campaign were included in the survey to serve as a control $[15,23]$. Process evaluation in Wave 4 involved a subset of participants ( 80 adults per county) responding to a phone survey asking if they had seen the clinic-based educational materials (posters, brochures) during the past year.

2.11. Statistical Analyses. The primary outcome was being within guidelines for CRC screening at the end of each intervention period (as determined by MRR) and the time point of interest was Wave 4 . Because $18 \%$ of the participants could not have their CRC screening status confirmed through a medical record, their screening status was imputed using a linear mixed model containing random county effects and fixed effects of predictors whose association with baseline screening status was significant at the 0.25 level or better as reported in Paskett et al. [16]. For each of the 50 imputed data sets, the proportion screened at Wave 1 and 4 was computed for each county. An ANCOVA model weighted by the inverse of the theoretical variance of the Wave 4 cluster means [24] was then used to compare Wave 4 screening rates between the intervention and comparison groups adjusting for Wave 1 screening rates and the results were combined across imputed data sets. As a sensitivity analysis, ANCOVA modeling was repeated for subjects whose medical record confirmed CRC screening status was observed (i.e., complete case analysis) and using self-reported CRC screening status. The same methods were used to compare intervention and comparison at Waves 2 and 3.

Linear models weighted by the number of participants in each arm at Wave 4 were used to explore the effect of the intervention on whether they talked with their doctor about family history of CRC, changes in bowel habits, rectal bleeding, and having a CRC screening test. The association between several patient/doctor interaction variables assessed at Wave 4 and CRC screening were assessed using multivariable logistic regression. Each behavior was included in a model that adjusted for county, marital status, insurance status, gender, smoking status, education, and employment. All analyses were performed using SAS v9.2 and v9.3 (SAS Institute, Cary, NC). Imputations were performed using the MMI_IMPUTE SAS macro [25].

\section{Results}

The demographic characteristics of participants $(n=4,491)$ by study wave in the intervention and comparison counties are presented in Table 1.

3.1. CRC Screening Rates. The estimated screening rates by wave are presented in Figure 2. The rates presented are averages across 50 imputed data sets. Participants from intervention counties were slightly more likely to have been within guidelines at Waves 1 and 4 . However, after adjusting for baseline CRC screening rate, there was no difference in the Wave 4 screening rates between the intervention and comparison counties $(p=0.50)$. Wave 2 screening rates did not differ by treatment $\operatorname{arm}(p=0.74)$, while participants in the intervention counties were less likely to be screened at Wave 3 than participants in the comparison counties $(p=$ 0.02 ) controlling for the county-level rates in Wave 1.

\subsection{Participant-Reported Physician Actions regarding CRC} and CRC Screening. Of the 1,091 participants who completed the survey at Wave $4,39(6.9 \%)$ participants from the intervention counties and 40 (7.6\%) participants from the comparison counties reported that their doctor asked them 


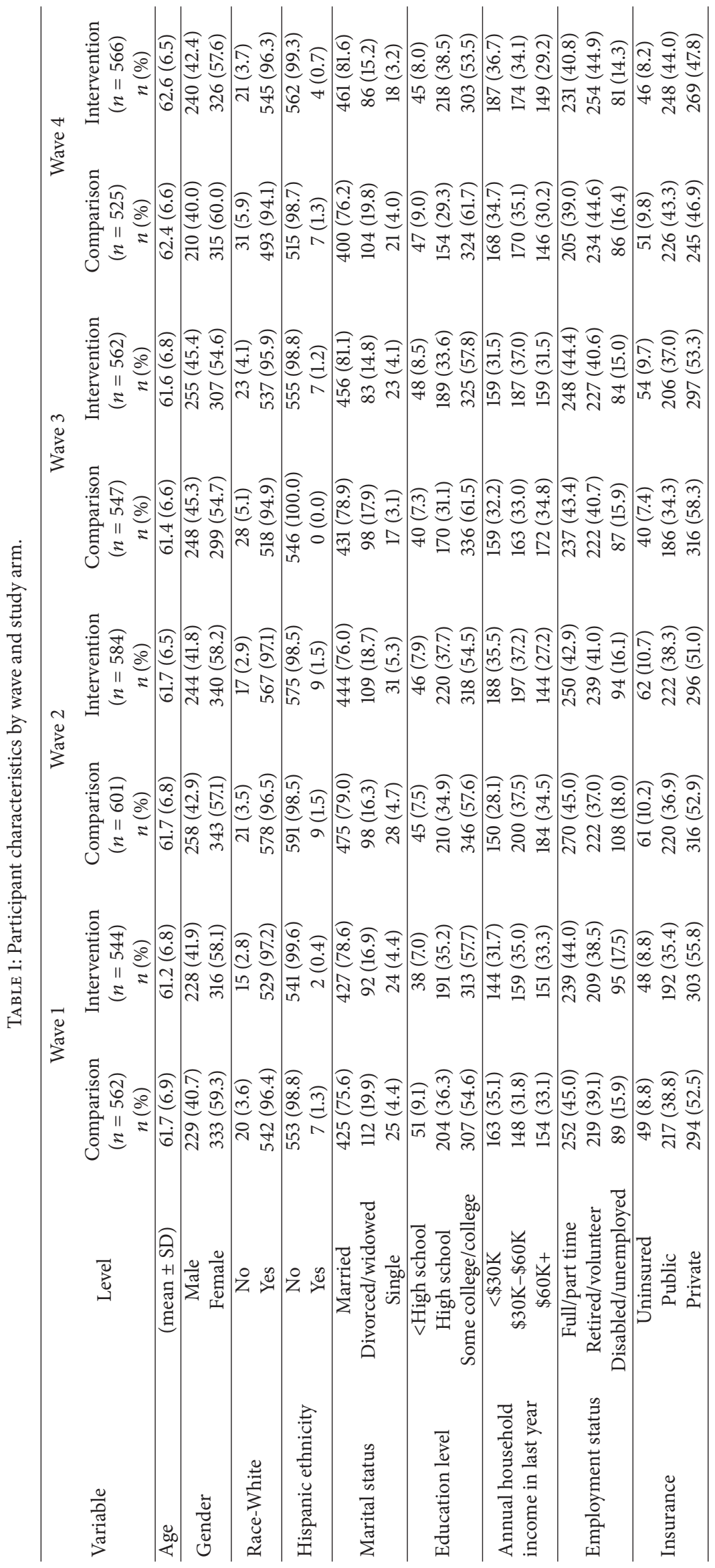




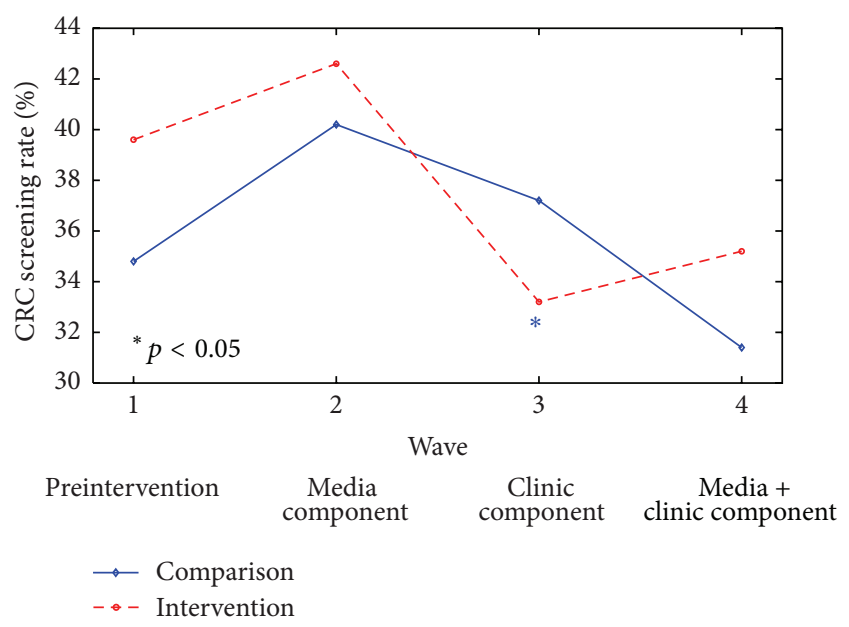

FIGURE 2: Unadjusted colorectal cancer (CRC) screening rates by intervention and comparison groups over time. Estimates are average over 50 imputed data sets.

about their family history of CRC ( $p=0.65)$. Fiftysix $(9.9 \%)$ participants from intervention counties and 76 (14.5\%) participants from comparison counties reported that their doctor asked about changes in their bowel habits $(p=0.009)$. A similar proportion of participants from intervention counties (8.1\%) and comparison counties (9.0\%) reported that they talked to their doctor about rectal bleeding $(p=0.64)$. One hundred ninety-two (33.9\%) participants from intervention counties and $172(32.8 \%)$ participants from comparison counties reported that they talked to their doctor about having a CRC screening test $(p=0.64)$. Lastly, a similar proportion of participants from intervention counties (85.1\%) and comparison counties (81.9\%) reported their willingness to have a CRC screening, if recommended by a doctor ( $p=$ 0.47 , adjusting for baseline rate).

3.3. Predictors of CRC Screening. In separate multivariable models adjusting for county and demographic data (age, gender, race, ethnicity, marital status, education level, income, employment, and insurance), perceived CRC risk, intention to screen, and physician actions regarding CRC and CRC screening were statistically significant predictors of being within guidelines for CRC screening at Wave 4 (Table 2). Specifically, high perceived risk of CRC (OR $=1.79,95 \%$ $\mathrm{CI}=1.08,2.95)$, willingness to have a CRC screening test if recommended by a doctor $(\mathrm{OR}=6.23,95 \% \mathrm{CI}=3.45,11.27)$, and not thinking about talking to their doctor about a test in the next year $(\mathrm{OR}=0.53,95 \% \mathrm{CI}=0.35,0.78)$ were associated with being within guidelines for CRC screening. Participants who asked their doctor for a CRC screening test $(\mathrm{OR}=1.96$, $95 \% \mathrm{CI}=1.13,3.38)$ and talked to their doctor about eating more fruits and vegetables $(\mathrm{OR}=1.47,95 \% \mathrm{CI}=1.07,2.03)$, family history of $\mathrm{CRC}(\mathrm{OR}=1.95,95 \% \mathrm{CI}=1.11,3.41)$, changes in bowel habits $(\mathrm{OR}=1.86,95 \% \mathrm{CI}=1.21,2.87)$, and having a CRC screening test $(\mathrm{OR}=1.82,95 \% \mathrm{CI}=1.34,2.46)$ were more likely to be within guidelines for CRC screening. Lastly, those participants whose doctors asked them to have a CRC screening test $(\mathrm{OR}=10.02,95 \% \mathrm{CI}=5.68,17.69)$ were more likely to be within guidelines for CRC screening.

3.4. Process Evaluation. After the media campaign (Wave 2), $14.3 \%$ of the 502 participants from intervention counties reported seeing the correct billboard encouraging CRC screening. Of the 507 participants from intervention counties who answered questions about seeing the study posters, $12.4 \%$ reported seeing at least one of the three correct CRC screening posters. Odds of CRC screening were not greater among participants who reported having seen the correct billboard versus those who did not $(\mathrm{OR}=0.87,95 \% \mathrm{CI}=0.51$ 1.50 ), nor were they greater among participants who reported having seen at least one of the correct posters $(\mathrm{OR}=1.42,95 \%$ $\mathrm{CI}=0.82-2.46$, resp.), versus those who did not.

After the combined media and clinic campaign (Wave 4), 978 participants (503 intervention and 475 comparison) who reported having visited a doctor in the past year answered questions about seeing the clinic-based educational materials. Of the 503 participants from intervention counties who reported having visited a doctor in the past year, 57.9\% reported seeing an ACS poster and 53.3\% reported seeing a brochure about CRC screening at the doctor's office. There was no effect of reporting having seen either an ACS poster $(\mathrm{OR}=1.34,95 \% \mathrm{CI}=0.90-2.01)$ or a brochure $(\mathrm{OR}=1.03,95 \%$ $\mathrm{CI}=0.69-1.52)$ on being within CRC screening guidelines.

\section{Discussion}

This group-randomized trial assessed the impact of a countylevel intervention, consisting of media and clinic components, to increase CRC screening among Ohio Appalachian adults. The findings indicate that, despite a high willingness to have CRC screening, the intervention did not have an effect on CRC screening among the adults in the intervention counties, as approximately $35 \%$ of the participants had completed a CRC screening test in both the intervention and comparison counties. This result is similar to previously reported rates among Appalachian residents [15, 26-28]. Significant predictors of CRC screening within guidelines among participants were high perceived risk of CRC, willingness to have a CRC test if recommended by a doctor, doctor recommendation of a CRC screening test, and patient-physician communication about changes in bowel habits, family history of CRC, and eating fruits and vegetables.

We considered a number of possible explanations for the null results, including low exposure to the intervention. Participants may not have visited the locations where the posters and billboards were displayed. Process evaluation indicated that participants from the intervention counties were exposed to the clinic-directed intervention (i.e., 52\% reported seeing the brochures); however, CRC screening rates did not differ between the two study groups suggesting that the media campaign and the clinic educational materials about CRC screening were not effective, as designed. Results of similar studies have raised questions about the efficacy of these types of interventions to bring sustained lifestyle 
TABLE 2: Multivariable logistic regression results for being within CRC screening guidelines (Wave 4) .

\begin{tabular}{lcc}
\hline Predictor & $\begin{array}{c}\text { OR for CRC screening } \\
(95 \% \text { CI })\end{array}$ & $\begin{array}{l}1.79(1.08,2.95) \\
\text { High perceived risk of CRC }\end{array}$ \\
Willingness to have a CRC screening test if recommended by doctor & $6.23(3.45,11.27)$ & 0.0233 \\
Thought about talking to doctor about completing a CRC screening & $0.53(0.35,0.78)$ & $<0.0001$ \\
test in the next year & $1.96(1.13,3.38)$ & $1.47(1.07,2.03)$ \\
Patient asked doctor for a CRC screening test & $1.95(1.11,3.41)$ & 0.0016 \\
Talked to doctor about eating more fruits and vegetables & $1.86(1.21,2.87)$ & 0.0160 \\
Talked to doctor about family history of CRC & $1.82(1.34,2.46)$ \\
Talked to doctor about changes in bowel habits & $10.02(5.68,17.69)$ \\
Talked to doctor about having a CRC screening test & 0.0199 \\
Doctor asked patient to have a CRC screening test & 0.0048 \\
\hline
\end{tabular}

${ }^{a}$ Each variable was considered individually after adjusting for county and demographic factors. Each line represents a separate model. Results are from 30 multiply imputed datasets.

changes and promote use of preventive health services [2931]. When applied to a lower SES population with challenges related to health care access $[9,24,28,32]$, perhaps this media and clinic intervention only raised consciousness and intention (as demonstrated by previous media campaigns [1113]) but could not lead to a major behavior change.

Future studies should consider utilizing media- and clinic-based interventions to increase knowledge and awareness of CRC screening coupled with personal contact from lay health advisors (LHAs) and patient navigators (PNs) to facilitate access to and completion of screening. LHA and $\mathrm{PN}$ interventions among underserved populations have been successful because they typically use trusted community members who understand the association between SES and cultural factors, as well as provider factors, associated with behavior change $[33,34]$. With this knowledge, they serve as a bridge between the community and health care system by providing information, support, and encouragement [33, 34]. Previous studies using LHA and PN intervention programs to promote cancer screening have found significantly increased CRC screening rates [34-37]. Modeling these coordinated, targeted, and community-based programs in Ohio Appalachia could be similarly successful.

4.1. Limitations. The current study had several limitations. First, the overall rates of CRC screening in the clinics during the study were not measured. This would provide information about the potential for increasing rates of CRC screening among specific clinic patients. Also, we did not survey physicians about their recommendations for CRC screening and perceived barriers to CRC screening faced by their patients. This study utilized cross-sectional data which can limit the comparability across study waves and prohibits insight into the impact of the intervention. It is possible that people who completed the surveys after the clinic-based intervention (i.e., Wave 3 ) could have seen Wave 2 intervention material, causing a bias in their response. However, the main outcome was the comparison between
Wave 4 and Wave 1 screening rates, and a cumulative effect of the interventions was expected. Lastly, study results may have limited generalizability because participants lived in one region of the US and were primarily non-Hispanic white.

\section{Conclusion}

This study tested the effectiveness of an intervention to increase rates of CRC screening among adults living in Ohio Appalachian counties. The county-level campaign consisted of media- (billboards, posters, and newspaper advertisements) and clinic-based (ACS brochures and posters) components about CRC and CRC screening. There were no differences in CRC screening rates between participants from the intervention and comparison counties at the end of the study, as measured by cross-sectional survey and MRR. Future research should examine how media- and clinic-based interventions can be modified to improve CRC screening rates among this underserved population.

\section{Conflict of Interests}

There is no actual, potential, or perceived conflict of interests by any of the authors.

\section{Acknowledgments}

The authors thank the Appalachian Community Cancer Network and the Appalachia Ohio Community Advisory Board for their contributions to this project. This study was supported by National Institutes of Health Grants 5R24MD002785-05 (P.I. EDP) and P30 CA016058 (OSU Comprehensive Cancer Center Behavioral Measurement Shared Resource). Jesse J. Plascak is supported by a grant from the National Cancer Institute Cancer Education Grant (R25CA092408). No other financial disclosures were reported by the authors of this paper. 


\section{References}

[1] American Cancer Society, Cancer Facts and Figures, 2014, American Cancer Society, Atlanta, Ga, USA, 2014.

[2] A. M. Cole, J. E. Jackson, and M. Doescher, "Colorectal cancer screening disparities for rural minorities in the United States," Journal of Primary Care \& Community Health, vol. 4, no. 2, pp. 106-111, 2013.

[3] H. M. Sineshaw, A. S. Robbins, and A. Jemal, "Disparities in survival improvement for metastatic colorectal cancer by race/ethnicity and age in the United States," Cancer Causes \& Control, vol. 25, no. 4, pp. 419-423, 2014.

[4] D. T. Liss and D. W. Baker, "Understanding current racial/ethnic disparities in colorectal cancer screening in the United States: the contribution of socioeconomic status and access to care," American Journal of Preventive Medicine, vol. 46, no. 3, pp. 228236, 2014.

[5] J. S. Haas, P. Brawarsky, A. Iyer, G. M. Fitzmaurice, B. A. Neville, and C. Earle, "Association of area sociodemographic characteristics and capacity for treatment with disparities in colorectal cancer care and mortality," Cancer, vol. 117, no. 18, pp. 4267-4276, 2011.

[6] R. L. Siegel, L. Sahar, A. Robbins, and A. Jemal, "Where can colorectal cancer screening interventions have the most impact?" Cancer Epidemiology Biomarkers \& Prevention, vol. 24, no. 8, pp. 1151-1156, 2015.

[7] Appalachian Community Cancer Network, The Cancer Burden in Appalachia, 2009.

[8] American Cancer Society, Ohio Cancer Facts and Figures, 2005, American Cancer Society Ohio Division, Columbus, Ohio, USA, 2005.

[9] R. Abramson and J. Haskell, Encyclopedia of Appalachia, University of Tennessee Press, 2006.

[10] B. Levin, D. A. Lieberman, B. McFarland et al., "Screening and surveillance for the early detection of colorectal cancer and adenomatous polyps, 2008: a joint guideline from the American Cancer Society, the US Multi-Society Task Force on Colorectal Cancer, and the American College of Radiology," CA: Cancer Journal for Clinicians, vol. 58, no. 3, pp. 130-160, 2008.

[11] C. Broadwater, J. Heins, C. Hoelscher, A. Mangone, and C. Rozanas, "Skin and colon cancer media campaigns in Utah," Preventing Chronic Disease, vol. 1, no. 4, article A18, 2004.

[12] L. Zittleman, C. Emsermann, M. Dickinson et al., "Increasing colon cancer testing in rural Colorado: evaluation of the exposure to a community-based awareness campaign," $B M C$ Public Health, vol. 9, article 288, 2009.

[13] B. H. Nguyen, S. J. McPhee, S. L. Stewart, and H. T. Doan, "Effectiveness of a controlled trial to promote colorectal cancer screening in Vietnamese Americans," American Journal of Public Health, vol. 100, no. 5, pp. 870-876, 2010.

[14] D. U. Ekwueme, D. H. Howard, C. A. Gelb, S. H. Rim, and C. P. Cooper, "Analysis of the benefits and costs of a national campaign to promote colorectal cancer screening: CDC's screen for life-national colorectal cancer action campaign," Health Promotion Practice, vol. 15, no. 5, pp. 750-758, 2014.

[15] M. L. Katz, P. Reiter, D. Fickle et al., "Community involvement in the development and feedback about a colorectal cancer screening media campaign in Ohio Appalachia," Health Promotion Practice, vol. 12, no. 4, pp. 589-599, 2011.

[16] E. D. Paskett, A. A. Llanos, G. S. Young, M. L. Pennell, C.-J. Lee, and M. L. Katz, "Correlates of colorectal cancer screening among residents of Ohio Appalachia," Journal of Community Health, vol. 38, no. 4, pp. 609-618, 2013.

[17] D. M. Murray, Design and Analysis of Group-Randomized Trials, Oxford University Press, New York, NY, USA, 1998.

[18] I. M. Rosenstock, "Historical origins of the health belief model," Health Education \& Behavior, vol. 2, no. 4, pp. 328-335, 1974.

[19] I. Ajzen and M. Fishbein, Understanding Attitudes and Predicting Social Behavior, Prentice-Hall, Englewood Cliffs, NJ, USA, 1980.

[20] A. Bandura, Social Foundations of Thought and Action: A Social Cognitive Theory, Prentice Hall, Englewood Cliffs, NJ, USA, 1986.

[21] A. Bandura, "A social cognitive approach to the exercise of control over AIDS infection," in Adolescents and AIDS: A Generation in Jeopardy, R. DiClemente, Ed., Sage Publications, Newbury Park, Calif, USA, 1992.

[22] R. H. Fazio, M. C. Powell, and C. J. Williams, "The role of attitude accessibility in the attitude-to-behavior process," Journal of Consumer Research, vol. 16, no. 3, pp. 280-288, 1989.

[23] S. S. Coughlin and T. D. Thompson, "Colorectal cancer screening practices among men and women in rural and nonrural areas of the United States, 1999," Journal of Rural Health, vol. 20, no. 2, pp. 118-124, 2004.

[24] J. L. Johnson, S. M. Kreidler, D. J. Catellier, D. M. Murray, K. E. Muller, and D. H. Glueck, "Recommendations for choosing an analysis method that controls Type I error for unbalanced cluster sample designs with Gaussian outcomes," Statistics in Medicine, 2015.

[25] S. A. Mistler, "A SAS macro for applying multiple imputation to multilevel data," in Proceedings of the SAS Global Forum, San Francisco, Calif, USA, April-May 2013, http://support.sas.com/ resources/papers/proceedings13/438-2013.pdf.

[26] J. L. Fisher, H. L. Engelhardt, J. A. Stephens et al., "Cancerrelated disparities among residents of Appalachia Ohio," Journal of Health Disparities Research and Practice, vol. 2, pp. 61-74, 2008.

[27] Appalachia Community Cancer Network, "The cancer burden in Appalachia," 2009, http://www.accnweb.com/.

[28] Y. N. Tarasenko and N. E. Schoenberg, "Colorectal cancer screening among rural Appalachian residents with multiple morbidities," Rural and Remote Health, vol. 11, no. 1, article 1553, 2011.

[29] M. Susser, "The tribulations of trials-intervention in communities," American Journal of Public Health, vol. 85, no. 2, pp. 156158, 1995.

[30] C. N. H. Jenkins, S. J. McPhee, J. A. Bird et al., "Effect of a media-led education campaign on breast and cervical cancer screening among Vietnamese-American women," Preventive Medicine, vol. 28, no. 4, pp. 395-406, 1999.

[31] S. A. Sabatino, B. Lawrence, R. Elder et al., "Effectiveness of interventions to increase screening for breast, cervical, and colorectal cancers: nine updated systematic reviews for the guide to community preventive services," American Journal of Preventive Medicine, vol. 43, no. 1, pp. 97-118, 2012.

[32] T. C. Davis, A. Rademaker, S. C. Bailey et al., "Contrasts in rural and urban barriers to colorectal cancer screening," American Journal of Health Behavior, vol. 37, no. 3, pp. 289-298, 2013.

[33] E. D. Paskett, J. M. McLaughlin, A. M. Lehman, M. L. Katz, C. M. Tatum, and J. M. Oliveri, "Evaluating the efficacy of lay health advisors for increasing risk-appropriate pap test screening: a randomized controlled trial among Ohio appalachian women," 
Cancer Epidemiology Biomarkers and Prevention, vol. 20, no. 5, pp. 835-843, 2011.

[34] S. Percac-Lima, R. W. Grant, A. R. Green et al., "A culturally tailored navigator program for colorectal cancer screening in a community health center: a randomized, controlled trial," Journal of General Internal Medicine, vol. 24, no. 2, pp. 211-217, 2009.

[35] C. L. Holt, M. S. Litaker, I. C. Scarinci et al., "Spiritually based intervention to increase colorectal cancer screening among African Americans screening and theory-based outcomes from a randomized trial," Health Education and Behavior, vol. 40, no. 4, pp. 458-468, 2013.

[36] T. T. Nguyen, M. B. Love, C. Liang et al., "A pilot study of lay health worker outreach and colorectal cancer screening among Chinese Americans," Journal of Cancer Education, vol. 25, no. 3, pp. 405-412, 2010.

[37] F. J. Feltner, G. E. Ely, E. T. Whitler, D. Gross, and M. Dignan, "Effectiveness of community health workers in providing outreach and education for colorectal cancer screening in Appalachian Kentucky," Social Work in Health Care, vol. 51, no. 5, pp. 430-440, 2012. 


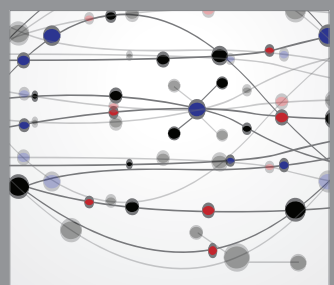

The Scientific World Journal
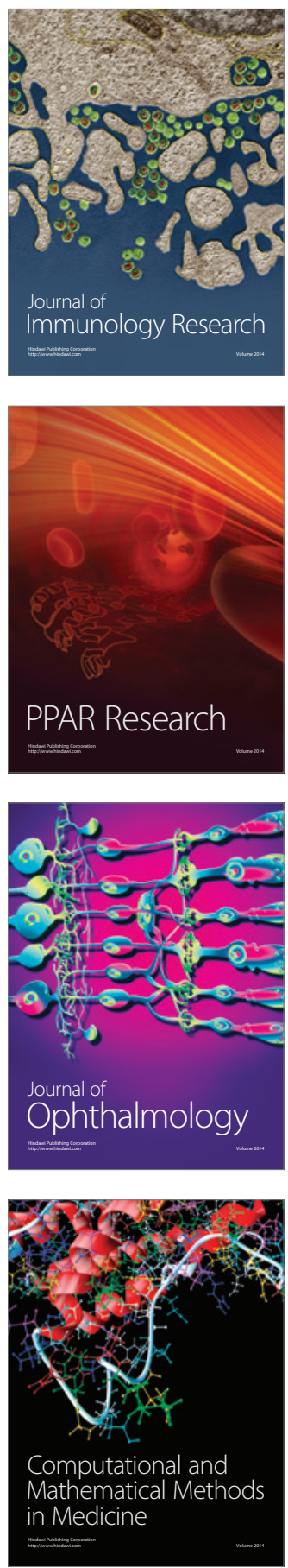

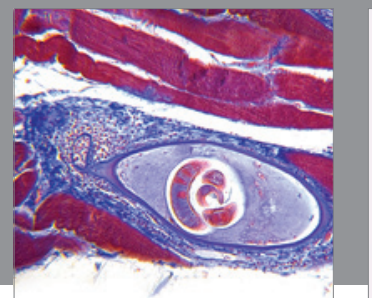

Gastroenterology

Research and Practice
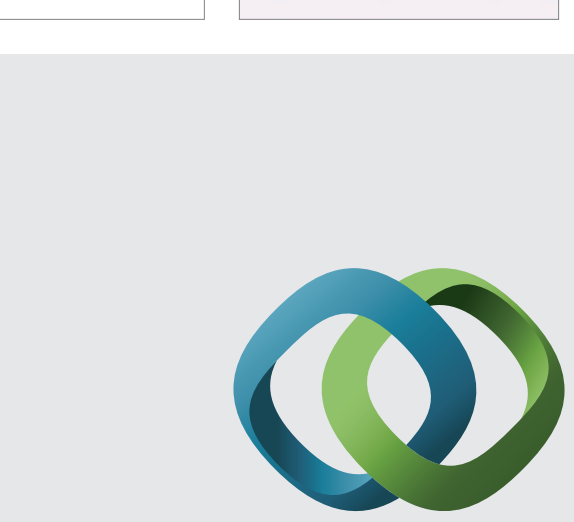

\section{Hindawi}

Submit your manuscripts at

http://www.hindawi.com
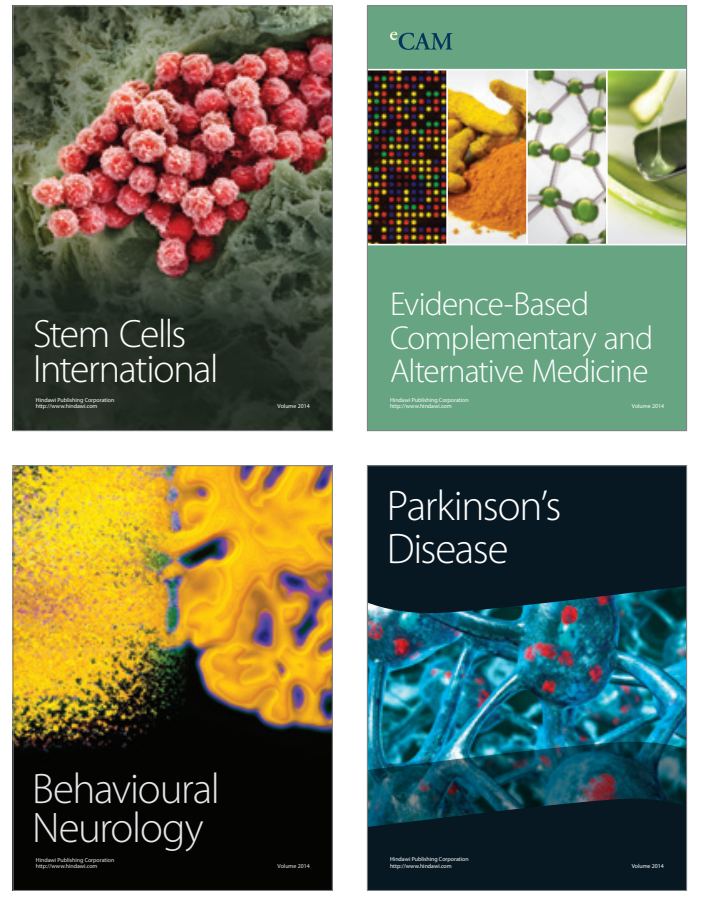
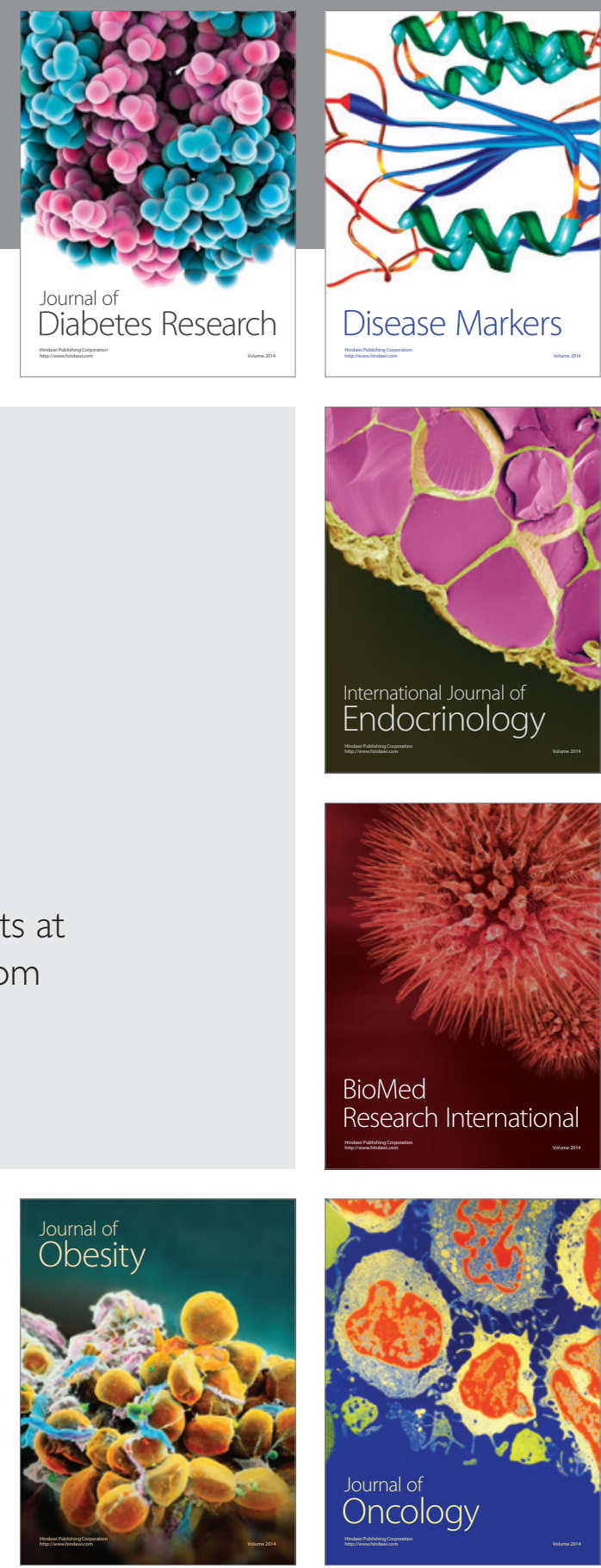

Disease Markers
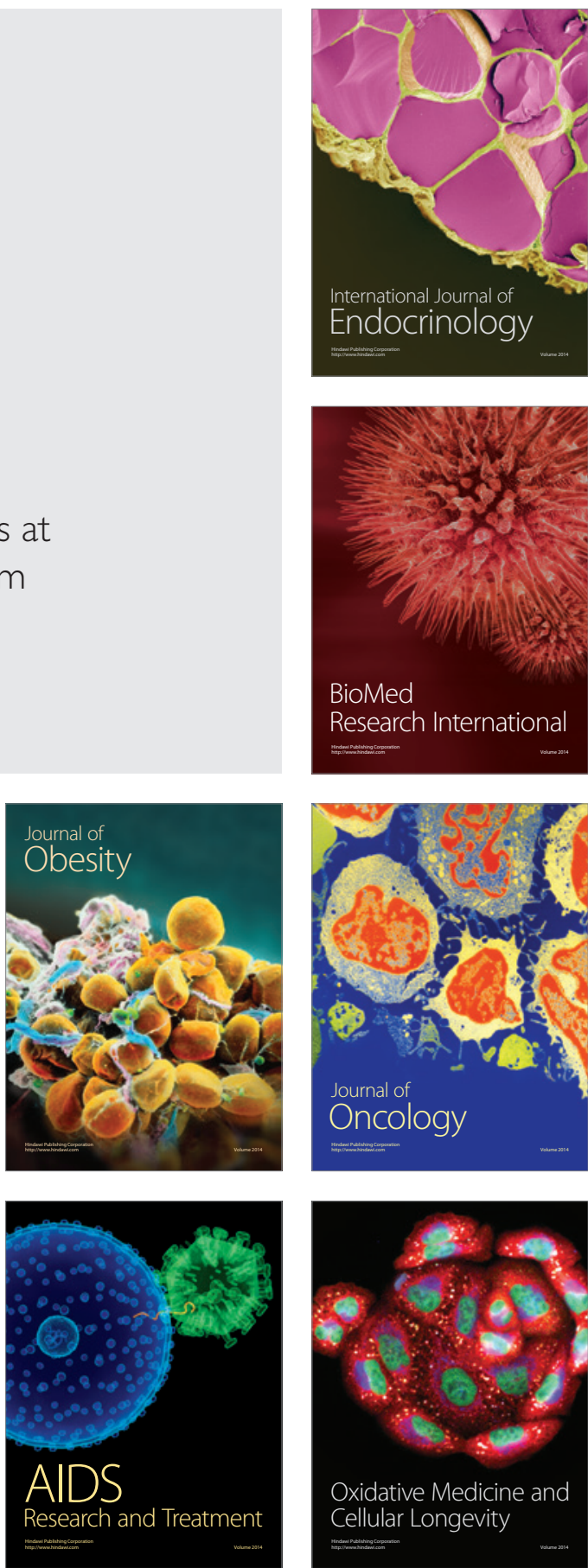